CUBO A Mathematical Journal

Vol.21, No-02, (77-98). August 2019

\title{
Certain results for $\eta$-Ricci Solitons and Yamabe Solitons on quasi-Sasakian 3-Manifolds
}

\author{
Sunil Kumar Yadav ${ }^{1}$, Abhishek Kushwaha ${ }^{2}$ and Dhruwa Narain ${ }^{3}$ \\ ${ }^{1}$ Department of Mathematics, \\ Poornima College of Engineering, \\ ISI-6,RIICO Institutional Area, Sitapura, Jaipur- 302022, Rajasthan,India \\ prof_sky16@yahoo.com \\ ${ }^{2,3}$ Department of Mathematics $\&$ Statistics \\ D.D.U.Gorakhpur University, \\ Gorakhpur-273009, Uttar Pradesh, India \\ ab11sk1991@gmail.com, dhruwanarain.dubey@gmail.com
}

\begin{abstract}
We classify quasi-Sasakian 3-manifold with proper $\eta$-Ricci soliton and investigate its geometrical properties. Certain results of Yamabe soliton on such manifold are also presented. Finally, we construct an example of non-existence of proper $\eta$-Ricci soliton on 3-dimensional quasi-Sasakian manifold to illustrate the results obtained in previous section of the paper.
\end{abstract}

\section{RESUMEN}

Clasificamos 3-variedades cuasi-Sasakianas con solitones $\eta$-Ricci propios e investigamos sus propiedades geométricas. Ciertos resultados sobre el solitón de Yamabe en dichas variedades también se presentan. Finalmente, construimos un ejemplo de la no existencia de solitones $\eta$-Ricci propios en una 3-variedad cuasi-Sasakiana para ilustrar los resultados contenidos en el artículo.

Keywords and Phrases: Quasi-Sasakian 3-manifold, infinitesimal contact transformation, $\eta$ Ricci soliton, Yamabe soliton.

2010 AMS Mathematics Subject Classification: 53C15, 53C40, 53D10. 


\section{Introduction}

In 1982, Hamilton [17 introduced the notion of Ricci flow to find a canonical metric on a smooth manifold. The Ricci flow is an evolution equation for metric $g_{i j}$ on a Riemannian manifold defined as follows:

$$
\frac{\partial g_{i j}}{\partial t}=-2 R_{i j}
$$

where $R_{i j}$ denotes the Ricci tensor of a Riemannian manifold and $t$ is the time. Ricci soliton are special solution of the Ricci flow equation (1.1) of the form $g_{i j}=\sigma(t) \Psi_{t} g_{i j}$ with the initial condition $g_{i j}(0)=g_{i j}$, where $\Psi_{t}$ is the diffeomorphisms of $M$ and $\sigma(t)$ is the scaling function. A Ricci soliton is a natural generalization of an Einstein metric. We recall the notion of Ricci soliton according to [9]. On a Riemannian manifold $M$, a Ricci soliton is a triple $(g, V, \mu)$ with the Riemannian metric $\mathrm{g}$, a vector field $\mathrm{V}$, called potential vector field, $\mu$ a real scalar and $S$ is the Ricci tensor such that

$$
\left(\mathfrak{L}_{V} g\right)(X, Y)+2 S(X, Y)+2 \mu g(X, Y)=0
$$

where $\mathfrak{L}_{V}$ is the Lie-derivative along the vector field $V$ on $M$. It is clear that a Ricci soliton with $V$ zero or a Killing vector field reduces to an Einstein metric. A Ricci soliton is said to be shrinking, steady and expanding according as $\mu$ is negative, zero and positive, respectively. The Ricci soliton have been studied by several authors such as ([12],[18, [20, ,28], [36]).

As a generalization of a Ricci soliton, the notion of $\eta$-Ricci soliton was introduced by Cho and Kimura [10]. This notion has also been studied in [10] for Hopf hypersurfaces in complex-spaceforms. An $\eta$-Ricci soliton is a 4-tuple $(g, V, \mu, \alpha)$, where $V$ is a vector field on $M, \mu$ and $\alpha$ are real constants and $\mathrm{g}$ is a Riemannian (or pseudo-Riemannian) metric satisfying the equation

$$
\left(\mathfrak{L}_{\vee g}\right)(X, Y)+2 S(X, Y)+2 \mu g(X, Y)+2 \alpha \eta(X) \eta(Y)=0
$$

where $S$ is the Ricci tensor associated to $\mathrm{g}$. In particular, if $\alpha=0$ then the notion of an $\eta$-Ricci soliton $(g, V, \mu, \alpha)$ reduces to the notion of a Ricci soliton $(g, V, \mu)$. If $\alpha \neq 0$, then the $\eta$-Ricci soliton are known as the proper $\eta$-Ricci soliton. Thus the notion of $\eta$-Ricci soliton have been studied by many authors like $([7,[8,[31,32$, ,33]). The notion of Yamabe flow was introduced by Richard Hamilton at the same time as the Ricci flow [17, as a tool for constructing metrics of constant scalar curvature in a given conformal class of Riemannian metrics on $\left(M^{n}, g\right)(n \geq 3)$. A timedependent metric $\mathrm{g}(\cdot, \mathrm{t})$ on a Riemannian or, pseudo Riemannian manifold $M$ is said to evolve by the Yamabe flow if the metric $\mathrm{g}$ satisfies

$$
\frac{\partial g(t)}{\partial t}=-\kappa g(t), \quad g(0)=g_{0}
$$

on $\mathrm{M}$, where $\mathrm{k}$ is the scalar curvature correspond to $\mathrm{g}$. Ye [35] has found that a point-wise elliptic gradient estimate for the Yamabe flow on a locally conformally flat compact Riemannian manifold. In case of Ricci flow, Yamabe soliton or the singularities of the Yamabe flow appear naturally. 
The significance of Yamabe flow lies in the fact that it is a natural geometric deformation to metric of constant scalar curvature. One notes that Yamabe flow corresponds to the fast diffusion case of the porous medium equation (the plasma equation) in mathematical physics. In dimension $n=2$, the Yamabe flow is equivalent to the Ricci flow (defined by $\frac{\partial}{\partial t} g(t)=-2 \alpha(t)$, where $\alpha$ stands for the Ricci tensor). However in dimension $n>2$, the Yamabe and Ricci flow do not agree, since the first one preserves the conformal class of metric but the Ricci flow does not in general. Just as Ricci soliton is a special solution of the Ricci flow, a Yamabe soliton is a special solution of the Yamabe flow that moves by one parameter family of diffeomorphism $\phi_{\mathrm{t}}$ generated by a fixed vector field $V$ on $M$, and homotheries, that is, $g(., t)=\sigma(t) \phi_{*}(t) g_{0}$.

A Yamabe soliton is defined on a Riemannian or, pseudo-Riemannian manifold ( $M, g$ ) by a vector field $\mathrm{V}$ satisfying the equation [].

$$
\frac{1}{2}\left(\mathfrak{L}_{V} g\right)(X, Y)=(\kappa-\lambda) g(X, Y)
$$

where $\mathfrak{L}_{V}$ denotes the Lie-derivative of the metric $g$ along the vector field $V, \kappa$ stands for the scalar curvature, while $\lambda$ is a soliton constant. A Yamabe soliton is said to be expanding, steady, or shrinking, respectively, if $\lambda<0, \lambda=0$ or $\lambda>0$. Otherwise, it will be called indefinite. Given a Yamabe soliton, if $V=D f$ holds for a smooth function $f: M \rightarrow \mathfrak{R}$ on $M$, the equation (1.5) becomes Hess $f=(r-\lambda) g$, where Hess $f$ denotes the Hessian of $f$ and $D$ denotes the gradient operator of $g$ on $M^{n}$. In this case $f$ is called the potential function of the Yamabe soliton and $g$ is said to be a gradient Yamabe soliton.

The notion of quasi-Sasakian structure was introduced by Blair [4] to unify Sasakian and cosymplectic structures. Tanno [30] also added some remarks on quasi-Sasakian structure.The properties of such manifold have been studied by several authors,viz., Gonzalez and Chinea [16], Kanemaki 21] and Oubina 26]. Kim [22] studied quasi-Sasakian manifold and proved that fibred Riemannian spaces with invariant fibers normal to the structure vector field do not admit nearly Sasakian or contact structure but a Sasakian or cosymplectic structure. Recently, quasi-Sasakian manifold have been the subject of growing interest in view of finding the significant of applications to physics, in particular to supargravity and magmatic theory ([3],1]). Quasi-Sasakian structure have wide application in the mathematical analysis of string theory ([2, 14] ). On a 3-dimensional quasi-Sasakian manifold, the structure function $\beta$ was defined by Olszak [27] and with the help of this function he has obtained necessary and sufficient conditions for the manifold to be conformally flat [25. Next he has proved that if the manifold is additionally conformally flat with $\beta=$ constant, then (a) it is locally a product of $R$ and a two-dimensional Kaehlerian space of constant Gauss curvature ( the cosymplectic case), or, (b) it is constant positive curvature ( the non-cosymplectic case, here the quasi-Sasakian structure is homothetic to a Sasakian structure).

Now, we give some necessary definition and proposition that are uses in latter section.

Definition1.1[6] A vector field $\mathrm{V}$ is said to be conformal for Yamabe soliton if it satisfying the 
equation

$$
\mathfrak{L}_{\vee g}=2 \omega g,
$$

where $\omega$ is called the conformal coefficient, that is, $\omega=(\kappa-\lambda)$. Moreover, if $\omega=0$, is equivalent to $\mathrm{V}$ being Killing.

Definition1.2 [8] A vector field $\mathrm{X}$ on an almost contact Riemannian manifold $\mathrm{M}$ is said to be infinitesimal transformation if there exists a smooth function $v$ on $\mathrm{M}$ such that

$$
\left(\mathfrak{L}_{X \eta}\right)(\mathrm{Y})=v \eta(Y)
$$

for every smooth vector field $\mathrm{X}$ and $\mathrm{Y}$. If $\mathrm{v}=0$ then $\mathrm{X}$ is called a strict infinitesimal transformation.

Proposition1.134 On an $\mathrm{n}$-dimensional Riemannian or, pseudo Riemannian manifold $\left(\mathrm{M}^{\mathrm{n}}, \mathrm{g}\right)$ endowed with a conformal vector field $\mathrm{V}$, we have

$$
\begin{gathered}
\left(\mathfrak{L}_{V} S\right)(X, Y)=-(n-2) g(\nabla \times D \omega, Y)+(\Delta \omega) g(X, Y), \\
\left(\mathfrak{L}_{V K}\right)=-2 \omega K+2(n-1) \Delta \omega,
\end{gathered}
$$

for any vector fields $\mathrm{X}$ and $\mathrm{Y}$, where $\mathrm{D}$ denotes the gradient operator and $\Delta=-\operatorname{div} \mathrm{D}$ denotes the Laplacan operator of $\mathrm{g}$.

The outline of this paper is to consider 3-dimensional quasi-Sasakian manifold with the structure function $\beta$ is constant. In Section 2, we recall the basic results of $\eta$-Ricci soliton on quasi-Sasakian 3-manifold. In Section 3 and Section 4, we examine the $\eta$-Ricci soliton on quasi-Sasakian 3-manifold admitting codazzi type and cyclic parallel Ricci tensor, respectively. Further, the Section 5, Section 6 and Section 7, deals with an almost pseudo Ricci symmetric, $\varphi$-Ricci symmetric and conformally flat with $\eta$-Ricci soliton on quasi-Ssakian 3- manifold respectively. The geometrical properties of a special weakly Ricci symmetric and $\eta$-recurrent on quasi-Sasakian 3-manifold are studied in Section 8 and Section 9, respectively. In Section 10, we deals quasi-Sasakian 3-manifolds with $\mathrm{Q} \cdot \mathrm{R}=0$ and obtain new results for $\eta$-Ricci soliton on such manifold. In Section 11, we deduce some results related to Yamabe soliton on quasi-Sasakian 3-manifold. At last, we construct an example of non-existence of proper $\eta$-Ricci soliton on quasi-Sasakian 3-manifold.

\section{Preliminaries}

Let $M$ be a $(2 n+1)$-dimensional an almost contact metric manifold equipped with an almost contact metric structure $(\varphi, \zeta, \eta, g)$ consisting of a $(1,1)$ tensor field $\varphi$, a vector field $\zeta$, a 1 -form $\eta$ and a Riemannian metric $\mathrm{g}$, which satisfies

$$
\varphi^{2}=-I+\eta \otimes \zeta,
$$




$$
\begin{array}{cl}
\eta(\zeta)=1, \quad \eta \circ \zeta=0, & \varphi \zeta=0, \\
g(\varphi \mathrm{U}, \varphi \mathrm{V})=\mathrm{g}(\mathrm{U}, \mathrm{V})-\eta(\mathrm{U}) \eta(\mathrm{V}), & \eta(\mathrm{U})=\mathrm{g}(\mathrm{U}, \zeta),
\end{array}
$$

for all $U, V \in \chi(M)$, where $\chi(M)$ is the Lie-algebra of the vector fields of $M^{2 n+1}$. Let $\Phi$ be the fundamental 2-form of $M^{2 n+1}$ defined by

$$
\Phi(\mathrm{U}, \mathrm{V})=\mathrm{g}(\mathrm{U}, \varphi \mathrm{V})
$$

for all $\mathrm{U}, \mathrm{V} \in \chi(M)$. Then $\Phi(\mathrm{U}, \zeta)=0, \mathrm{U} \in \chi(M) \cdot \mathrm{M}^{2 \mathrm{n}+1}$ is said to be quasi-Sasakian if the almost contact structure $(\varphi, \zeta, \eta, g)$ is normal and the fundamental 2-form $\Phi$ is closed, that is, every $U, V \in \mathfrak{I}^{2 n+1}$, where $\mathfrak{I}^{2 n+1}$ denotes the modulus of vector fields on $M^{2 n+1}$.

$$
\text { (i) }[\varphi, \varphi](\mathrm{U}, \mathrm{V})+\mathrm{d} \eta(\mathrm{U}, \mathrm{V}) \zeta=0, \quad \text { (ii) } \mathrm{d} \Phi=0 \text {. }
$$

There are many types of quasi-Sasakian structures ranging from the cosymplectic case, $d \eta=$ $O(\operatorname{rank} \eta=1)$, to the Sasakian case, $\eta \wedge(d \eta)^{n} \neq 0(\operatorname{rank} \eta=2 n+1, \Phi=d \eta)$. The 1 -form $\eta$ has $\operatorname{rank} \bar{r}=2 p$ if $(d \eta)^{p} \neq 0$ and $\eta \wedge(d \eta)^{p}=0$, and has $\operatorname{rank} \bar{r}=2 p+1$ if $(d \eta)^{p+1}=0$ and $\eta \wedge(d \eta)^{p} \neq 0$. We also say that $\bar{r}$ is the rank of the quasi-Sasakian structure. Blair [7, proved that there are no quasi-Sasakian structure of even rank, some theorems regarding Kaehlerian manifolds and existence of quasi-Sasakian manifold.

An almost contact metric manifold $\mathrm{M}^{2 \mathrm{n}+1}$ is a 3-dimensional quasi-Sasakian manifold if and only if [27]

$$
\nabla \mathrm{u} \zeta=-\beta \varphi \mathrm{U}, \mathrm{u} \in \chi(\mathrm{M}),
$$

for a certain function $\beta$ on $M$, such that $\zeta \beta=0, \nabla$ being the operator of the covariant differentiation with respect to the Levi-Civita connection on $M$. Clearly, such a quasi-Sasakian manifold is cosymplectic if and only if $\beta=0$. Here we have shown that the assumption $\zeta \beta=0$ is not necessary.

As per consequence (2.6), we find that 27 .

$$
(\nabla \mathrm{u} \varphi)(\mathrm{V})=\beta[\mathrm{g}(\mathrm{U}, \mathrm{V}) \zeta-\eta(\mathrm{V}) \mathrm{U}] .
$$

In view of $(2.6)$ and $(2.7)$, we obtain

$$
\nabla_{\mathrm{u}}\left(\nabla_{\mathrm{V}} \zeta\right)=-(\mathrm{U} \beta) \varphi \mathrm{V}-\beta^{2}[\mathrm{~g}(\mathrm{U}, \mathrm{V}) \zeta-\eta(\mathrm{V}) \mathrm{U}]-\beta \varphi \nabla \mathrm{u} \mathrm{V} .
$$

This implies that

$$
\mathrm{R}(\mathrm{U}, \mathrm{V}) \zeta=-(\mathrm{U} \beta) \varphi \mathrm{V}+(\mathrm{V} \beta) \varphi \mathrm{U}+\beta^{2}[\eta(\mathrm{V}) \mathrm{U}-\eta(\mathrm{U}) \mathrm{V}]
$$

Thus from (2.9), we get 


$$
\mathrm{R}(\mathrm{U}, \mathrm{V}, \mathrm{W}, \zeta)=(\mathrm{U} \beta) \mathrm{g}(\varphi \mathrm{V}, \mathrm{W})-(\mathrm{V} \beta) \mathrm{g}(\varphi \mathrm{U}, \mathrm{W})-\beta^{2}[\eta(\mathrm{V}) \mathrm{g}(\mathrm{U}, \mathrm{W})-\eta(\mathrm{U}) \mathrm{g}(\mathrm{V}, \mathrm{W})]
$$

Substituting $\mathrm{U}=\zeta$ in $(2.10)$, we have

$$
R(\zeta, V, W, \zeta)=\beta^{2}[g(V, W)-\eta(V) \eta(W)]+g(\varphi V, W) \zeta \beta
$$

Interchanging $\mathrm{V}$ and $\mathrm{W}$ of (2.11) it yields

$$
R(\zeta, W, V, \zeta)=\beta^{2}[g(W, V)-\eta(W) \eta(V)]+g(\varphi W, V) \zeta \beta
$$

Since $R(\zeta, V, W, \zeta)=R(W, \zeta, \zeta, V)=R(\zeta, W, V, \zeta)$. Then from (2.11) and(2.12), we obtain

$$
[\mathrm{g}(\varphi \mathrm{V}, \mathrm{W})-\mathrm{g}(\varphi \mathrm{W}, \mathrm{V})] \zeta \beta=0 .
$$

Therefore, we can easily verify that $\zeta \beta=0$.

In a 3-dimensional Riemannian manifold we have

$$
\begin{aligned}
\mathrm{R}(\mathrm{U}, \mathrm{V}) \mathrm{W}= & \{\mathrm{S}(\mathrm{V}, \mathrm{W}) \mathrm{U}-\mathrm{S}(\mathrm{U}, \mathrm{W}) \mathrm{V}+\mathrm{g}(\mathrm{V}, \mathrm{W}) \mathrm{QU}-\mathrm{g}(\mathrm{U}, \mathrm{W}) \mathrm{QV}\} \\
& -\frac{\mathrm{K}}{2}\{\mathrm{~g}(\mathrm{~V}, \mathrm{~W}) \mathrm{U}-\mathrm{g}(\mathrm{U}, \mathrm{W}) \mathrm{V}\}
\end{aligned}
$$

where $\mathrm{S}$ and $\mathrm{k}$ are the Ricci tensor and the scalar curvature, respectively, and $\mathrm{Q}$ denotes the Ricci operator defined by $\mathrm{g}(\mathrm{Qu}, \mathrm{V})=\mathrm{S}(\mathrm{U}, \mathrm{V})$.

It is well known that the Ricci tensor S of a quasi-Sasakian 3-manifold is given by [28]

$$
\mathrm{S}(\mathrm{U}, \mathrm{V})=\left[\frac{\kappa}{2}-\beta^{2}\right] \mathrm{g}(\mathrm{U}, \mathrm{V})+\left[3 \beta^{2}-\frac{\kappa}{2}\right] \eta(\mathrm{U}) \eta(\mathrm{V})
$$

As a consequence of (2.15), we find the Ricci operator $\mathrm{Q}$

$$
\mathrm{QU}=\left[\frac{\kappa}{2}-\beta^{2}\right] \mathrm{U}+\left[3 \beta^{2}-\frac{\kappa}{2}\right] \eta(\mathrm{U}) \zeta \text {. }
$$

From (2.15), we obtain

$$
\mathrm{S}(\mathrm{U}, \zeta)=2 \beta^{2} \eta(\mathrm{U})
$$

Keeping in mind the Equ.(2.12),(2.13),(2.14) and (2.15), we have

$$
\mathrm{R}(\mathrm{U}, \mathrm{v}) \zeta=\beta^{2}[\eta(\mathrm{V}) \mathrm{U}-\eta(\mathrm{u}) \mathrm{V}]
$$

for all $\mathrm{U}, \mathrm{V}, \in \chi(\mathrm{M})$. Also from (2.6), we have

$$
(\nabla \mathrm{u}) \mathrm{V}=\mathrm{g}\left(\nabla_{\mathrm{u}} \zeta, \mathrm{V}\right)=-\beta \mathrm{g}(\varphi \mathrm{U}, \mathrm{V})
$$

Again from (2.15), it follows that

$$
\mathrm{S}(\varphi \mathrm{U}, \varphi \mathrm{V})=\mathrm{S}(\mathrm{U}, \mathrm{V})-2 \beta^{2} \eta(\mathrm{U}) \eta(\mathrm{V})
$$


Proposition 2.1. A 3-dimensional non-cosymplectic quasi-Sasakian manifold with $\eta$-Ricci soliton is an $\eta$-Einstein manifold.

Proof. Assume that the quasi-Sasakian 3-manifold admits a proper $\eta$-Ricci soliton $(\mathrm{g}, \zeta, \mu, \alpha)$. Then from (1.3), we have

$$
2 \mathrm{~S}(\mathrm{U}, \mathrm{V})=-\left(\mathrm{L}_{\zeta} \mathrm{g}\right)(\mathrm{U}, \mathrm{V})-2 \mu \mathrm{g}(\mathrm{U}, \mathrm{V})-2 \alpha \eta(\mathrm{U}) \eta(\mathrm{V})
$$

for all smooth vector fields $\mathrm{U}, \mathrm{V} \in \chi(\mathrm{M})$. Of the two natural situations regarding the vector field $V: V \in \operatorname{span}\{\zeta\}$ and $V \perp \zeta$, we investigate only the case $V=\zeta$. Our interest is in the expression for $L_{\zeta} g+2 S+2 \mu g+2 \alpha \eta \otimes \eta$.

A straight forward computations give

$$
\begin{aligned}
\left(\mathrm{L}_{\zeta} \mathrm{g}\right)(\mathrm{U}, \mathrm{V}) & =\mathrm{g}\left(\nabla_{\mathrm{u}} \zeta, \mathrm{V}\right)+\mathrm{g}\left(\mathrm{U}, \nabla_{\mathrm{V}} \zeta\right), \\
& =-\beta[\mathrm{g}(\varphi \mathrm{U}, \mathrm{V})+\mathrm{g}(\mathrm{U}, \varphi \mathrm{V})]=0 .
\end{aligned}
$$

Using (2.22) in (2.21), we get

$$
S(U, V)=-\mu g(U, V)-\alpha \eta(U) \eta(V) .
$$

From the last equation, the proof ends.

Proposition 2.2. If a 3-dimensional non-cosymplectic quasi-Sasakian manifold admits $\eta$-Ricci soliton, then $\mu+\alpha=-2 \beta^{2}$.

Proof. From (2.15), we have

$$
S(U, V)=\left[\frac{K}{2}-\beta^{2}\right] g(U, V)+\left[3 \beta^{2}-\frac{\kappa}{2}\right] \eta(U) \eta(V) .
$$

Comparing (2.24) with (2.23), we get $\mu=\frac{1}{2}\left[2 \beta^{2}-\kappa\right]$ and $\alpha=\frac{1}{2}\left[\kappa-6 \beta^{2}\right]$. In continuation we get $\mu+\alpha=-2 \beta^{2}$. From the last equation, the proof ends.

\section{Ricci tensor of Codazzi type}

Gray [15] introduced the notion of cyclic parallel and Codazzi type Ricci tensors. A Riemannian manifold is said to possesses a cyclic parallel Ricci tensor if its non-vanishing Ricci tensor $S$ of type $(0,2)$ satisfies the condition

$$
\left(\nabla_{\mathrm{u}} \mathrm{S}\right)(\mathrm{V}, \mathrm{W})+\left(\nabla_{\mathrm{V}} \mathrm{S}\right)(\mathrm{W}, \mathrm{U})+\left(\nabla_{\mathrm{W}} \mathrm{S}\right)(\mathrm{U}, \mathrm{V})=0
$$


for arbitrary vector fields $\mathrm{U}, \mathrm{V}, \mathrm{W}$ on $\mathrm{M}$. Again a Riemannian manifold is said to have a Ricci tensor of Codazzi type if $S$ is non-zero and satisfies

$$
\left(\nabla_{\mathrm{u}} \mathrm{S}\right)(\mathrm{V}, \mathrm{W})=\left(\nabla_{\mathrm{V}} \mathrm{S}\right)(\mathrm{U}, \mathrm{W})
$$

for all the vector fields $\mathrm{U}, \mathrm{V}, \mathrm{W}$ on $\mathrm{M}$.

We consider proper $\eta$-Ricci soliton on quasi-Sasakian 3-manifold with Ricci tensor of Codazzi type. Taking covariant derivative of (2.23) along $W$ and using (2.19), we have

$$
\begin{aligned}
\left(\nabla_{W} S\right)(\mathrm{U}, \mathrm{V}) & =-\alpha\left[\left(\nabla_{W} \eta\right)(\mathrm{U}) \eta(\mathrm{V})+\eta(\mathrm{U})\left(\nabla_{W} \eta\right)(\mathrm{V})\right] \\
& =\alpha \beta[\mathrm{g}(\mathrm{U}, \varphi \mathrm{W}) \eta(\mathrm{V})+\mathrm{g}(\mathrm{V}, \varphi \mathrm{W}) \eta(\mathrm{U})] .
\end{aligned}
$$

Since the Ricci tensor $S$ of $M$ is of Codazzi type. Then

$$
\left(\nabla_{\mathrm{W}} \mathrm{S}\right)(\mathrm{U}, \mathrm{V})=\left(\nabla_{\mathrm{u}} \mathrm{S}\right)(\mathrm{W}, \mathrm{V})
$$

Making use of (3.3) in (3.4), we yields

$$
\alpha \beta[g(U, \varphi W) \eta(V)+g(V, \varphi W) \eta(U)]=\alpha \beta[g(W, \varphi U) \eta(V)+g(V, \varphi U) \eta(W)] .
$$

Setting $W=\zeta$ in (3.5), we theorize $\beta \neq 0, \alpha=0$, which is a refutation. Thus a non-cosymlectic quasi-Sasakian 3-manifold with a Ricci tensor of Codazzi type does not admits a proper $\eta$-Ricci soliton. In this way we terminate the following result:

Theorem 3.1. A non-cosymplectic quasi-Sasakian 3-manifold accompanied by Ricci tensor of Codazzi type does not possess a proper $\eta$-Ricci soliton.

Corollary 3.2. For a proper $\eta$-Ricci soliton on a non-cosymplectic quasi-Sasakian 3-manifold, the scalar curvature is constant if and only if the vector field $\zeta$ is harmonic.

Corollary 3.3. There exists no constant scalar curvature for a proper $\eta$-Ricci soliton of noncosymplectic quasi-Sasakian 3-manifold, provided the vector field $\zeta$ is non-harmonic.

\section{Cyclic parallel Ricci tensor}

This section is affectionate to the study of proper $\eta$-Ricci soliton on quasi-Sasakian 3-manifold bearing cyclic parallel Ricci tensor. On that account

$$
\left(\nabla_{\mathrm{u}} \mathrm{S}\right)(\mathrm{V}, \mathrm{W})+\left(\nabla_{\mathrm{V}} \mathrm{S}\right)(\mathrm{W}, \mathrm{U})+\left(\nabla_{\mathrm{W}} \mathrm{S}\right)(\mathrm{U}, \mathrm{V})=0
$$

On the other hand, we have (3.3) and left hand side of (4.1), we have

$$
\begin{gathered}
\left(\nabla_{\mathrm{u}} \mathrm{S}\right)(\mathrm{V}, \mathrm{W})+\left(\nabla_{\mathrm{V}} \mathrm{S}\right)(\mathrm{W}, \mathrm{U})+\left(\nabla_{\mathrm{W}} \mathrm{S}\right)(\mathrm{U}, \mathrm{V})=\alpha \beta[\mathrm{g}(\mathrm{V}, \varphi \mathrm{U}) \eta(\mathrm{W})+\mathrm{g}(\mathrm{W}, \varphi \mathrm{U}) \eta(\mathrm{V})+\mathrm{g}(\mathrm{W}, \varphi \mathrm{V}) \eta(\mathrm{U}) \\
+\mathrm{g}(\mathrm{U}, \varphi \mathrm{V}) \eta(\mathrm{W})+\mathrm{g}(\mathrm{U}, \varphi \mathrm{W}) \eta(\mathrm{V})+\mathrm{g}(\mathrm{V}, \varphi \mathrm{W}) \eta(\mathrm{U})]
\end{gathered}
$$


Taking in hand (2.3) and (4.2), we reached

$$
\left(\nabla_{\mathrm{u}} \mathrm{S}\right)(\mathrm{V}, \mathrm{W})+\left(\nabla_{\mathrm{V}} \mathrm{S}\right)(\mathrm{W}, \mathrm{U})+\left(\nabla_{\mathrm{W}} \mathrm{S}\right)(\mathrm{U}, \mathrm{V})=0
$$

Thus we are in condition to plight the following result:

Theorem 4.1. A quasi-Sasakian 3-manifold bearing proper $\eta$-Ricci soliton always satisfies cyclic parallel Ricci tensor.

\section{$5 \quad$ Almost pseudo Ricci symmetric}

Chaki and Kawaguchi [11] introduced the concept of almost pseudo Ricci symmetric manifolds as an extended class of pseudo symmetric manifolds. A Riemannian manifold $(M, g)$ is called an almost pseudo Ricci symmetric manifold $(A P R S)_{n}$, if its Ricci tensor $S$ of type $(0,2)$ is not identically zero and satisfying the following condition:

$$
\left(\nabla_{\mathrm{u}} \mathrm{S}\right)(\mathrm{V}, \mathrm{W})=[\mathrm{A}(\mathrm{U})+\mathrm{B}(\mathrm{U})] \mathrm{S}(\mathrm{V}, \mathrm{W})+\mathrm{A}(\mathrm{V}) \mathrm{S}(\mathrm{U}, \mathrm{W})+\mathrm{A}(\mathrm{W}) \mathrm{S}(\mathrm{U}, \mathrm{V}),
$$

where $A$ and $B$ are two non-zero 1-forms defined by

$$
A(U)=g\left(U, \rho_{1}\right), \quad B(U)=g\left(U, \rho_{2}\right) .
$$

By taking cyclic sum of (5.1), we see that

$$
\begin{gathered}
\left(\nabla_{\mathrm{u}} \mathrm{S}\right)(\mathrm{V}, \mathrm{W})+\left(\nabla_{\mathrm{V}} \mathrm{S}\right)(\mathrm{W}, \mathrm{U})+\left(\nabla_{\mathrm{W}} \mathrm{S}\right)(\mathrm{U}, \mathrm{V})=[3 \mathrm{~A}(\mathrm{U})+\mathrm{B}(\mathrm{U})] \mathrm{S}(\mathrm{V}, \mathrm{W}) \\
+[3 \mathrm{~A}(\mathrm{~V})+\mathrm{B}(\mathrm{V})] \mathrm{S}(\mathrm{U}, \mathrm{W})+[3 \mathrm{~A}(\mathrm{~W})+\mathrm{B}(\mathrm{W})] \mathrm{S}(\mathrm{U}, \mathrm{V})
\end{gathered}
$$

Let $M$ admits a cyclic Ricci tensor, then (5.3) reduces

$$
\begin{aligned}
{[3 A(U)} & +B(U)] S(V, W)+[3 A(V)+B(V)] S(U, W) \\
& +[3 A(W)+B(W)] S(U, V)=0
\end{aligned}
$$

Replacing $W$ by $\zeta$ in (5.4) and using (2.23) and (5.2), we get

$$
\begin{gathered}
-(\mu+\alpha)[3 A(U)+B(U)] \eta(V)-(\mu+\alpha)[3 A(V)+B(V)] \eta(U) \\
+\left[3 \eta\left(\rho_{1}\right)+\eta\left(\rho_{2}\right)\right] S(U, V)=0 .
\end{gathered}
$$

In (5.5), substituting $\mathrm{V}=\zeta$ and using (2.2), (2.23) and (5.2), we yield

$$
-(\mu+\alpha)[3 A(U)+B(U)]-2(\mu+\alpha)\left[3 \eta\left(\rho_{1}\right)+\eta\left(\rho_{2}\right)\right] \eta(U)=0 .
$$

Again replacing $\mathrm{U}$ by $\zeta$ and using (5.2) in (5.6), we obtain

$$
-(\mu+\alpha)\left[3 \eta\left(\rho_{1}\right)+\eta\left(\rho_{2}\right)\right]=0
$$


which implies

$$
3 \eta\left(\rho_{1}\right)+\eta\left(\rho_{2}\right)=0,
$$

since $(\mu+\alpha) \neq 0$. In view of (5.6) and (5.8), it follows that $3 A(U)+B(U)=0$. Thus we can state the following result.

Theorem 5.1. There is no almost pseudo Ricci symmetric proper $\eta$-Ricci soliton on non-cosymplectic quasi-Sasakian 3-manifold admitting cyclic Ricci tensor, unless 3A+B vanishes everywhere on $\mathrm{M}$.

Consequently, if we keep in mind from (5.7) that $3 \eta\left(\rho_{1}\right)+\eta\left(\rho_{2}\right) \neq 0$, in this case $\mu+\alpha=0$, but for $\eta$-Ricci soliton on non-cosymplectic quasi-Sasakian 3-manifold $\alpha+\mu=-2 \beta^{2}$. Therefore for this condition $\alpha=-\beta^{2}$ and $\mu=-\beta^{2}$. Thus we state the following result.

Corollary 5.2. A proper $\eta$-Ricci soliton on almost pseudo Ricci symmetric non-cosymplectic quasi-Sasakian 3-manifold with cyclic Ricci tensor is of type $\left(\mathrm{g}, \mathrm{V},-\beta^{2}\right.$, $\left.-\beta^{2}\right)$.

\section{$6 \quad \varphi$-Ricci Symmetric}

This segment is affectionate to the study of $\varphi$-Ricci Symmetric proper $\eta$-Ricci soliton on a quasiSasakian 3-manifold and deduce some result. A quasi-Sasakian 3-manifold is said to be $\varphi$-Ricci symmetric if the Ricci operator Q satisfies

$$
\varphi^{2}(\nabla \mathrm{uQ}) \mathrm{V}=0,
$$

for all smooth vector fields $U, V \in \chi(M)$. If $X, Y$ are orthogonal to $\zeta$, then the manifold is said to be locally $\varphi$-Ricci symmetric. It is well-known that $\varphi$-symmetric implies $\varphi$-Ricci symmetric, but the converse, is not, in general true. $\varphi$-Ricci symmetric Sasakian manifolds have been studied by De and Sarkar [13].

From (2.23), it follows that

$$
\mathrm{QU}=-\mu \mathrm{U}-\alpha \eta(\mathrm{U}) \zeta,
$$

for all smooth vector fields U. Proceeding covariant derivative of (6.2), we acquire

$$
\begin{aligned}
(\nabla \mathrm{uQ}) \mathrm{V} & =-\alpha \beta[\mathrm{g}(\mathrm{U}, \varphi \mathrm{V}) \zeta-\eta(\mathrm{V}) \varphi \mathrm{U}] \\
& =\alpha \beta[\mathrm{g}(\varphi \mathrm{U}, \mathrm{V}) \zeta+\eta(\mathrm{V}) \varphi \mathrm{U}] .
\end{aligned}
$$

Applying $\varphi^{2}$ on both sides of (6.3), we get

$$
\varphi^{2}(\nabla \mathrm{uQ}) \mathrm{V}=\alpha \beta \eta(\mathrm{V}) \varphi^{3} \mathrm{U}
$$


Making use of (6.1), from (6.4), it walk behind that $\beta \neq 0, \alpha=0$, which is a counter statement. Thus we are in a condition to plight the following result:

Theorem 6.1. A $\varphi$-Ricci symmetric non-cosymplectic quasi-Sasakian 3-manifold does not admits a proper $\eta$-Ricci soliton.

In 24, authors prove that in a 3-dimensional non-cosymplectic quasi-Sasakian mnaifold $\varphi$ Ricci symmetric and $\varphi$-symmetric are equivalent provided $\beta$ is a constant. Thus using this facts we state the following result.

Corollary 6.2. A $\varphi$-symmetric non-cosymplectic quasi-Sasakian 3-manifold does not possess a proper $\eta$-Ricci soliton.

Differentiating (2.16) covariantly along $W$ and applying $\varphi^{2}$ both side, we get

$$
\varphi^{2}\left(\nabla_{W} \mathrm{Q}\right) \mathrm{V}=\frac{1}{2}\left[\mathrm{~d} \kappa(\mathrm{W})(-\mathrm{V}+\eta(\mathrm{V}) \zeta)+\left(6 \beta^{2}-\kappa\right) \eta(\mathrm{V}) \varphi^{2}\left(\nabla_{W} \zeta\right)\right] .
$$

If $\mathrm{V}$ is orthogonal to $\zeta$, then from (6.4) and (6.5), we have

$$
\frac{1}{2} \mathrm{~d} \kappa(W) \mathrm{V}=0 .
$$

It implies that $d \kappa=0$. Hence the scalar curvature $\kappa$ is constant. Thus we state the following result.

Corollary 6.3. A non-cosymplectic quasi-Sasakian 3-manifold bearing proper $\eta$-Ricci soliton is locally $\varphi$-Ricci symmetric if and only if the scalar curvature $\mathrm{K}$ is constant.

\section{Conformally flat}

In this constituent we review conformally flat quasi-Sasakian 3-manifolds with a proper $\eta$-Ricci soliton. Then we have [25].

$$
\left(\nabla_{\mathrm{u}} \mathrm{S}\right)(\mathrm{V}, \mathrm{W})-\left(\nabla_{\mathrm{V}} \mathrm{S}\right)(\mathrm{U}, \mathrm{W})=\frac{1}{4}[\mathrm{~g}(\mathrm{~V}, \mathrm{~W}) \mathrm{d} \kappa(\mathrm{U})-\mathrm{g}(\mathrm{U}, \mathrm{W}) \mathrm{d} \kappa(\mathrm{V})]
$$

Making use of (2.23) in (7.1), we have

$$
\begin{array}{r}
\alpha \beta[g(V, \varphi \mathrm{U}) \eta(W)+g(W, \varphi \mathrm{U}) \eta(\mathrm{V})-\mathrm{g}(\mathrm{U}, \varphi \mathrm{V}) \eta(\mathrm{W})-\mathrm{g}(\mathrm{W}, \varphi \mathrm{V}) \eta(\mathrm{U})] \\
=\frac{1}{4}\left[\mathrm{~g}(\mathrm{~V}, \mathrm{~W}) \mathrm{d}_{\kappa}(\mathrm{U})-\mathrm{g}(\mathrm{U}, \mathrm{W}) \mathrm{d} \kappa(\mathrm{V})\right] .
\end{array}
$$

Substituting $\mathrm{U}=\zeta$ in (7.2), we obtain

$$
4 \alpha \beta g(\varphi \mathrm{W}, \mathrm{V})=-\eta(W) d \kappa(V) .
$$


That restricted to

$$
4 \alpha \beta \varphi \mathrm{V}=-\mathrm{d} \kappa(\mathrm{V}) \zeta \text {. }
$$

From the above equation it walk behind that $4 \alpha \beta \varphi^{2} \mathrm{~V}=0$ and hence $\beta \neq 0, \alpha=0$, which is a counter statement. Therefore we state the following result:

Theorem 7.1. A conformally flat non-cosymplectic quasi-Sasakian 3-manifold does not possess a proper $\eta$-Ricci soliton.

\section{Special weakly Ricci symmetric}

The notion of a special weakly Ricci symmetric manifold was introduced and studied by Singh and Quddus [29]. An $n$-dimensional Riemannian manifold $(M, g)$ is called a special weakly Ricci symmetric manifold $(\mathrm{SWRS})_{\mathrm{n}}$ if

$$
\left(\nabla_{X} S\right)(Y, Z)=2 \varepsilon(X) S(Y, Z)+\varepsilon(Y) S(X, Z)+\varepsilon(Z) S(Y, X)
$$

where $\varepsilon$ is a 1 -form and is defined by

$$
\varepsilon(X)=g(X, \rho),
$$

where $\rho$ is the associated vector field. Let the Eq.(8.1) and (8.2) hold on quasi-Sasakian 3-manifold. Taking cyclic sum of (8.1), we get

$$
\begin{aligned}
\left(\nabla_{X} S\right)(Y, Z) & +\left(\nabla_{Y} S\right)(Z, X)+\left(\nabla_{Z} S\right)(X, Y) \\
= & 4[\varepsilon(X) S(Y, Z)+\varepsilon(Y) S(Z, X)+\varepsilon(Z) S(X, Y)] .
\end{aligned}
$$

Let $M$ admits a cyclic parallel Ricci tensor. Then 8.3 reduces to

$$
\varepsilon(X) S(Y, Z)+\varepsilon(Y) S(Z, X)+\varepsilon(Z) S(X, Y)=0 .
$$

Taking $Z=\zeta$ in (8.4) and using (2.23) and (8.2), we have

$$
-(\alpha+\mu)[\varepsilon(X) \eta(Y)+\varepsilon(Y) \eta(X)]+\eta(\rho) S(X, Y)=0 .
$$

Again, taking $Y=\zeta$ in (8.5) and then using (2.23) and (8.2), we get

$$
-(\alpha+\mu)[\varepsilon(X)+2 \eta(\rho) \eta(X)]=0 .
$$

Taking $X=\zeta$ in (8.6) and using (8.2), we obtain

$$
-3(\alpha+\mu) \eta(\rho)=0 .
$$

In this case if $\eta(\rho)=0$ and $\alpha+\mu \neq 0$, then from (8.6) we have $\varepsilon(X)=0, \forall X \in \chi(M)$. Again if $\eta(\rho) \neq 0, \alpha+\mu=0$, in this case $\alpha=-\beta^{2}, \mu=-\beta^{2}$. It leads to the following result: 
Theorem 8.1. If a special weakly Ricci symmetric non-cosymplectic quasi-Sasakian 3-manifold with a proper $\eta$-Ricci soliton admits a cyclic parallel Ricci tensor, then the 1-form $\varepsilon$ is vanish identically on $\mathrm{M}$.

Corollary 8.2. A proper $\eta$-Ricci soliton on a special weakly Ricci symmetric non-cosymplectic quasi-Sasakian 3-manifold admits cyclic Ricci tensor is of type $\left(\mathrm{g}, \mathrm{V},-\beta^{2},-\beta^{2}\right)$ if the 1 -form $\varepsilon \neq 0$.

Again, if a complete Einstein quasi-Sasakian 3-manifold is compact. Then we have [19].

$$
S(X, Y)=\vartheta g(X, Y), \quad \vartheta=2 \beta^{2} .
$$

It is well-known that for complete Einstein quasi-Sasakian 3-manifold, $\left(\nabla_{X} S\right)(Y, Z)=0$ and $S(X, Y)=\vartheta g(X, Y)$. Then (8.1) gives

$$
2 \varepsilon(X) g(Y, Z)+\varepsilon(Y) g(X, Z)+\varepsilon(Z) g(Y, X)=0 .
$$

Taking $Z=\zeta$ in (8.8) and then using (8.2), we get

$$
2 \varepsilon(X) \eta(Y)+\varepsilon(Y) \eta(X)+\eta(\rho) g(Y, X)=0 .
$$

Again taking $X=\zeta$ in (8.9) and then using (8.2), we get

$$
3 \eta(\rho) \eta(Y)+\varepsilon(Y)=0 .
$$

Taking $Y=\zeta$ in (8.10) and using (8.2), we obtain

$$
\eta(\rho)=0
$$

Making use of (8.11) in (8.10), we get $\varepsilon(Y)=0, \forall Y \in \chi(M)$. Finally we have the following result:

Theorem 8.3. A special weakly Ricci symmetric non-cosymplectic quasi-Sasakian 3-manifold can not be compact if the 1 -form $\varepsilon \neq 0$.

\section{9 ฤ-recurrent}

A quasi-Sasakian manifold is said to be $\eta$-recurrent if its non-vanishing Ricci tensor $\mathrm{S}$ satisfies the following condition

$$
(\nabla \mathrm{u} S)(\varphi \mathrm{V}, \varphi \mathrm{W})=\mathrm{A}(\mathrm{U}) \mathrm{S}(\varphi \mathrm{V}, \varphi \mathrm{W}),
$$

for all $U, V, W \in \chi(M)$, where $A(U)=g(U, \rho), \rho$ is the associated vector field of the 1 -form $A$. In particular, if the 1 -form $A$ vanishes identically on $M$, then it is said to be $\eta$-parallel. This notion for Sasakian manifold was first introduced by Kon [21]. In view of (2.3), (2.19) and (2.23), we have

$$
\begin{array}{r}
(\nabla \mathrm{uS})(\varphi \mathrm{V}, \varphi \mathrm{W})=\mu \beta[\mathrm{g}(\mathrm{U}, \varphi \mathrm{V}) \eta(\mathrm{W})+\mathrm{g}(\mathrm{U}, \varphi \mathrm{W}) \eta(\mathrm{V}) \\
-\mathrm{g}(\mathrm{U}, \varphi \mathrm{W}) \eta(\mathrm{V})-\mathrm{g}(\varphi \mathrm{V}, \mathrm{U}) \eta(\mathrm{W})]=0 .
\end{array}
$$


Making use of (9.2) in (9.1), we get

$$
\mathrm{A}(\mathrm{U}) \mathrm{S}(\varphi \mathrm{V}, \varphi \mathrm{W})=0 .
$$

Again using (2.23) in (9.3), we obtain

$$
-\mu \mathrm{A}(\mathrm{U}) \mathrm{g}(\varphi \mathrm{V}, \varphi \mathrm{W})=0 .
$$

This implies that $A(U) \neq 0, g(\varphi V, \varphi W) \neq 0$. Therefore, we conclude that $\mu=0$, that is, the Ricci soliton is always steady. So we have the following result.

Theorem 9.1. If a non-cosymplectic quasi-Sasakian 3-manifold with proper $\eta$-Ricci soliton satisfying $\eta$-recurrent, then the Ricci soliton is always steady.

Corollary 9.2. The necessary condition for a non-cosymplectic quasi-Sasakian 3-manifold with proper $\eta$-Ricci soliton to be $\eta$-parallel, the Ricci soliton is always shrinking.

\section{The curvature condition $Q \cdot R=0$.}

In this section we are going to study, a proper $\eta$-Ricci soliton on a quasi-Sasakian 3-manifold that satisfying the curvature condition $Q \cdot R=0$. Then

$$
(\mathrm{Q} \cdot \mathrm{R})(\mathrm{U}, \mathrm{V}) \mathrm{W}=0
$$

for all smooth vector fields $U, V, W \in \chi(M)$. From (10.1), it is obvious that

$$
\mathrm{Q}(\mathrm{R}(\mathrm{U}, \mathrm{V}) \mathrm{W})-\mathrm{R}(\mathrm{QU}, \mathrm{V}) \mathrm{W}-\mathrm{R}(\mathrm{U}, \mathrm{QV}) \mathrm{W}-\mathrm{R}(\mathrm{U}, \mathrm{V}) \mathrm{QW}=0 .
$$

Making use of (2.14) and (2.23), Eq. (10.2) reduces to

$$
\begin{aligned}
4 \alpha \mu \eta & (\mathrm{U}) \eta(\mathrm{W}) \mathrm{V}+2 \mu^{2} \eta(\mathrm{U}) \eta(\mathrm{W}) \mathrm{V}-4 \alpha \mu \eta(\mathrm{V}) \eta(\mathrm{W}) \mathrm{U}+\alpha \kappa \eta(\mathrm{U}) \eta(\mathrm{W}) \mathrm{V} \\
& -\alpha \kappa \eta(\mathrm{V}) \eta(\mathrm{W}) \mathrm{U}+2 \mu[\{-\mu \mathrm{g}(\mathrm{V}, \mathrm{W})-\alpha \eta(\mathrm{V}) \eta(\mathrm{W})\} \mathrm{U} \\
& -\{-\mu \mathrm{g}(\mathrm{U}, \mathrm{W})-\alpha \eta(\mathrm{U}) \eta(\mathrm{W})\} \mathrm{V}+\mathrm{g}(\mathrm{V}, \mathrm{W})\{(-\mu \mathrm{U}-\alpha \eta(\mathrm{U}) \zeta\} \\
& -\mathrm{g}(\mathrm{U}, \mathrm{W})\left\{(-\mu \mathrm{V}-\alpha \eta(\mathrm{V}) \zeta\}-\frac{\kappa}{2}\{\mathrm{~g}(\mathrm{~V}, \mathrm{~W}) \mathrm{U}-\mathrm{g}(\mathrm{U}, \mathrm{W}) \mathrm{V}\}\right] \\
& -2 \alpha^{2} \eta(\mathrm{V}) \eta(\mathrm{W}) \mathrm{U}+\alpha \mu \mathrm{g}(\mathrm{V}, \mathrm{W}) \eta(\mathrm{U}) \zeta+\alpha^{2} \mathrm{~g}(\mathrm{~V}, \mathrm{~W}) \eta(\mathrm{U}) \zeta=0 .
\end{aligned}
$$

Putting $\mathrm{U}=\mathrm{W}=\zeta$ in (10.3), we get

$$
\begin{aligned}
4 \alpha \mu \mathrm{V} & +2 \mu^{2} \mathrm{~V}-4 \alpha \mu \eta(\mathrm{V}) \zeta+\alpha \kappa \mathrm{V}-\alpha \kappa \eta(\mathrm{V}) \zeta \\
& +2 \mu[-\mu \eta(\mathrm{V}) \zeta-\alpha \eta(\mathrm{V}) \zeta+\mu \mathrm{V}+\alpha \mathrm{V}-\mu \eta(\mathrm{V}) \zeta \\
& \left.-\alpha \eta(\mathrm{V}) \zeta+\mu \mathrm{V}+\alpha \eta(\mathrm{V}) \zeta-\frac{\kappa}{2} \eta(\mathrm{V}) \zeta+\frac{\mathrm{k}}{2}(\mathrm{~V})\right] \\
& -2 \alpha^{2} \eta(\mathrm{V}) \zeta+\mu \alpha \eta(\mathrm{V}) \zeta+\alpha^{2} \eta(\mathrm{V}) \zeta=0
\end{aligned}
$$


Applying the inner product of the above equation with $\zeta$, we obtain

$$
\alpha(\mu+\alpha) \eta(V)=0
$$

It walk behind that $\alpha \neq 0$, which is a counter statement. Thus $\alpha+\mu=0$. On other hand for a $\eta$ Ricci soliton on a quasi-Sasakian 3-manifold, $\alpha+\mu=-2 \beta^{2}$. Therefore for this condition $\alpha=-\beta^{2}$ and $\mu=-\beta^{2}$. Thus we sate the following result:

Theorem 10.1. A proper $\eta-$ Ricci soliton on a non-cosymplectic quasi-Sasakian 3-manifold satisfying the curvature condition $\mathrm{Q} \cdot \mathrm{R}=0$ is of type $\left(\mathrm{g}, \mathrm{V},-\beta^{2},-\beta^{2}\right)$.

As the dissertation of our work, we keep in mind the Corollary [5.2, Corollary 8.2 and Theorem 10.1. we state the following result.

Theorem 10.2. If a proper $\eta$-Ricci soliton on a non-cosymplectic quasi-Sasakian 3-manifold $M$ is of type $\left(\mathrm{g}, \mathrm{V},-\beta^{2},-\beta^{2}\right)$, then the following conditions are equivalent:

i) $\mathrm{M}^{\mathrm{n}}$ is almost pseudo Ricci symmetric with cyclic Ricci tensor,

ii) $\mathrm{M}^{\mathrm{n}}$ is special weakly Ricci symmetric and its Ricci tensor is cyclic parallel,

iii) $\mathrm{Q} \cdot \mathrm{R}=0$ holds on $\mathrm{M}^{\mathrm{n}}$.

\section{Yamabe solitons}

In this section we find some results related to Yamabe soliton on quasi-Sasakian 3-manifolds. We consider a Yamabe soliton $(\mathrm{g}, \zeta)$. From $(1.5)$ we have

$$
\frac{1}{2}\left(\mathfrak{L}_{\vee} \mathrm{g}\right)(X, Y)=(\kappa-\lambda) g(X, Y),
$$

which implies that

$$
g\left(\nabla_{X} \zeta, Y\right)+g\left(X, \nabla_{Y} \zeta\right)=2(\kappa-\lambda) g(X, Y) .
$$

Keeping in mind (2.6), Equ.(11.2) reduces to

$$
2(\kappa-\lambda) g(X, Y)=0
$$

Taking $X=\zeta$ in (11.3), we get $\lambda=\kappa$. Then equation (1.5) reduces to $\mathfrak{L}_{\vee} g=0$, that is, $V$ is Killing vector field. Moreover, $\lambda$ is constant then the scalar curvature $k$ is also constant. Thus we state the following result.

Theorem 11.1. If the metric of a 3-dimensional non-cosymplectic quasi-Sasakian 3-manifold is a Yamabe soliton then the manifold is space of constant curvature. 
Besides it, from (1.5), we have $\mathfrak{L}_{\vee} g=0$, thus $\mathrm{V}$ is Killing. Differentiating covariently along an arbitrary vector field $X$, we have $\nabla_{X} \mathfrak{L}_{V} g=0$.

The identity

$$
\left(\nabla_{\mathrm{X}} \mathfrak{L}_{V} \mathrm{~g}\right)(\mathrm{U}, \mathrm{W})=\mathrm{g}\left(\left(\mathfrak{L}_{V} \nabla\right)(\mathrm{X}, \mathrm{U}), \mathrm{W}\right)+\mathrm{g}\left(\left(\mathfrak{L}_{V} \nabla\right)(\mathrm{X}, \mathrm{W}), \mathrm{U}\right),
$$

can be reduced from the formula 34 .

$$
\left(\mathfrak{L}_{V} \nabla_{X} g-\nabla_{X} \mathfrak{L}_{V} g-\nabla_{[V, X]} g\right)(\mathrm{U}, \mathrm{W})=-\mathrm{g}\left(\left(\mathfrak{L}_{V} \nabla\right)(\mathrm{X}, \mathrm{U}), \mathrm{W}\right)-\mathrm{g}\left(\left(\mathfrak{L}_{V} \nabla\right)(\mathrm{X}, \mathrm{W}), \mathrm{U}\right) .
$$

This implies that

$$
\mathrm{g}\left(\left(\mathfrak{L}_{V} \nabla\right)(\mathrm{W}, \mathrm{X}), \mathrm{U}\right)+\mathrm{g}\left(\left(\mathfrak{L}_{V} \nabla\right)(\mathrm{W}, \mathrm{U}), \mathrm{X}\right)=0
$$

According to equation (11.4) and (11.5), the skew-symmetric property of $\phi$, we get $\left(\mathfrak{L}_{V} \nabla\right)(\mathrm{U}, \mathrm{W})=$ 0 , which implies that $\left(\mathfrak{L}_{V} \nabla\right)(\zeta, \zeta)=0$. Also, using geodesic properties of $\zeta$, we have

$$
\left(\mathfrak{L}_{\mathrm{V}} \nabla\right)(\mathrm{X}, \mathrm{U})=-\nabla_{\mathrm{X}} \nabla_{\mathrm{u}} \mathrm{V}-\nabla_{\nabla_{\mathrm{x}} \mathrm{u}} \mathrm{V}+\mathrm{R}(\mathrm{V}, \mathrm{X}) \mathrm{U},
$$

which yields $\nabla_{\zeta} \nabla_{\zeta} \mathrm{V}+\mathrm{R}(\mathrm{V}, \zeta) \zeta=0$. This means that $\mathrm{V}$ is Jacobi along the direction of $\zeta$. So we have the following result.

Theorem 11.2. If the metric of a non-cosymplectic quasi-Sasakian 3-manifold is a Yamabe soliton, then the flow vector field $\mathrm{V}$ is Killing and is Jacobi along the direction of $\zeta$.

It is well-known that the Reeb vector field $\zeta$ is a unit vector field, that is, $g(\zeta, \zeta)=1$.Taking Lie-derivative of it along the vector fled $\mathrm{V}$ and using (1.5), we get

$$
\eta\left(\mathfrak{L}_{\bigvee} \zeta\right)=-\left(\mathfrak{L}_{\bigvee} \eta\right)(\zeta)=(\lambda-\kappa) .
$$

Moreover, in view of $\omega=(\kappa-\lambda),(n=3)$ and Proposition 1.1, we obtain

(i) $\left(\mathfrak{L}_{\bigvee} S\right)(X, Y)=-g\left(\nabla_{X} D \kappa, Y\right)+\Delta \kappa g(X, Y)$.

(ii) $\left(\mathfrak{L}_{V K}\right)=-2 \kappa(\kappa-\lambda)+4 \Delta \kappa$.

Since $\mathrm{g}$ is a Yamabe soliton, then taking the Lie-derivative of (2.15), and using the above equation, we get

$$
\begin{aligned}
-g\left(\Delta_{X} D \kappa, Y\right)= & \frac{1}{2}\left(\mathfrak{L}_{V \kappa}\right)[g(X, Y)-\eta(X) \eta(Y)] \\
& +\left[2\left(\frac{\kappa}{2}-\beta^{2}\right)(\kappa-\lambda)\right] g(X, Y) \\
& +\left(3 \beta^{2}-\frac{\kappa}{2}\right)\left[\left(\mathfrak{L}_{V \eta}\right)(X) \eta(Y)+\left(\mathfrak{L}_{\vee} \eta(Y) \eta(X)\right] .\right.
\end{aligned}
$$


Since $\zeta$ is Killing, therefore $\zeta \kappa=0$. Differentiating covariantly along the direction of an arbitrary vector field $X$, we have $g\left(\Delta_{X} D \kappa, \zeta\right)=(\beta \varphi X) \kappa$. Substituting $Y=\zeta$ in above equation, we have

$$
-2 \beta(\varphi X) \kappa=\left[2\left(\kappa-2 \beta^{2}\right)(\kappa-\lambda)-2 \Delta \kappa\right] \eta(X)+\left(6 \beta^{2}-\kappa\right)[(\mathfrak{L} \bigvee \eta X+(\kappa-\lambda) \eta(X)] .
$$

Taking $X=\zeta$ in (11.9), using (11.6) and Proposition 1.1, we obtain

$$
\Delta \kappa=4 \beta^{2}(\kappa-\lambda)
$$

In view of (11.9) and(11.10), we yields

$$
\left(6 \beta^{2}-\kappa\right)(\mathfrak{L} \bigvee \eta) X=-2 \beta(\varphi X) \kappa-\left[(\kappa-\lambda)\left(\kappa-6 \beta^{2}\right] \eta(X)\right.
$$

Since $k$ is constant then from (11.11) one can say that either $\kappa=6 \beta^{2}$ or $\kappa \neq 6 \beta^{2}$. In particular if $\kappa=6 \beta^{2}$ then from (2.15) we have $S=2 \beta^{2} \mathrm{~g}$, that is, $M$ is an Einstein manifold of constant curvature $\beta^{2}$. Thus as per above consequences, we state the following result.

Corollary 11.3. If the metric of a 3-dimensional non-cosymplectic quasi-Sasakian manifold admits a Yamabe soliton and $\mathrm{\kappa}=6 \beta^{2}$ then the manifold is an Einstein.

Corollary 11.4. For a 3-dimensional cosymplectic manifold which admits a Yamabe soliton always has constant harmonic scalar curvature, that is $\Delta \kappa=0$.

Corollary 11.5. If a 3-dimensional non-cosymplectic quasi-Sasakian manifold with constant harmonic scalar curvature admitting Yamabe soliton then the manifold is space of constant curvature.

On the other hand, if $\kappa \neq 6 \beta^{2}$ then from (11.8), we get $\mathfrak{L}_{V \eta}=0$. Then the equation (1.7) implies that $v=0$. Thus we state the following result.

Theorem 11.6. If the metric of a 3-dimensional non-cosymplectic quasi-Sasakian manifold is a Yamabe soliton, then the conformal contact transformation of the conformal vector field is strict.

\section{An Example}

We consider a 3-dimensional manifold $M^{3}=\left\{(u, v, w) \in \mathfrak{R}^{3},(u, v, w) \neq(0,0,0)\right\}$, where $(u, v, w)$ is the standard coordinate in $\mathfrak{R}^{3}$. Let $\left(e_{1}, e_{2}, e_{3}\right)$ be linearly independent vector fields at each point of $M$, identify by

$$
e_{1}=\frac{\partial}{\partial v}, \quad e_{2}=\frac{\partial}{\partial w}, \quad e_{3}=\beta\left(\frac{\partial}{\partial u}+v \frac{\partial}{\partial w}-w \frac{\partial}{\partial v}\right)
$$

and

$$
\left[e_{1}, e_{2}\right]=0, \quad\left[e_{1}, e_{3}\right]=\beta e_{2}, \quad\left[e_{2}, e_{3}\right]=-\beta e_{1} .
$$


Let the Riemannian metric $g$ on $M^{3}$ is defined as

$$
g\left(e_{1}, e_{2}\right)=g\left(e_{2}, e_{3}\right)=g\left(e_{1}, e_{3}\right)=0, g\left(e_{1}, e_{1}\right)=g\left(e_{2}, e_{2}\right)=g\left(e_{3}, e_{3}\right)=1 .
$$

and given by

$$
g=\frac{1}{\beta^{2}}\left[\left(1-\beta^{2} v^{2}-\beta^{2} w^{2}\right) d u \otimes d u+\beta^{2} d v \otimes d v+\beta^{2} d w \otimes d w\right] .
$$

Let $\eta$ be the 1 -form has the significance

$$
\eta(U)=g\left(u, e_{3}\right)
$$

for any $\mathrm{U} \in \chi\left(\mathrm{M}^{3}\right)$ and $\varphi$ be the $(1,1)$ tensor field defined by

$$
\varphi e_{1}=-e_{2}, \quad \varphi e_{2}=e_{1}, \quad \varphi e_{3}=0 .
$$

Making use of the linearity of $\varphi$ and $g$, we have

$$
\begin{gathered}
\eta\left(e_{3}\right)=1, \\
\varphi^{2}(\mathrm{U})=-\mathrm{U}+\eta(\mathrm{U}) e_{3}
\end{gathered}
$$

and

$$
\mathrm{g}(\varphi \mathrm{U}, \varphi \mathrm{V})=\mathrm{g}(\mathrm{U}, \mathrm{V})-\eta(\mathrm{U}) \eta(\mathrm{V})
$$

for any $\mathrm{U}, W \in \chi\left(\mathrm{M}^{3}\right)$. Thus for $e_{3}=\zeta$, the structure $\left(M^{3}, \eta, \zeta, \varphi, g\right)$ leads to a contact metric structure on $M^{3}$. The Riemannian connection $\nabla$ of metric tensor $g$ is given by the beauty of Koszul's formula

$$
\begin{aligned}
2 \mathrm{~g}(\nabla \mathrm{u} V, W) & =\mathrm{U}(\mathrm{g}(\mathrm{V}, \mathrm{W}))+\mathrm{V}(\mathrm{g}(\mathrm{W}, \mathrm{X}))-\mathrm{W}(\mathrm{g}(\mathrm{U}, \mathrm{V})) \\
& -\mathrm{g}(\mathrm{U},[\mathrm{V}, \mathrm{W}])-\mathrm{g}(\mathrm{V},[\mathrm{U}, \mathrm{W}])+\mathrm{g}(\mathrm{W},[\mathrm{U}, \mathrm{V}])
\end{aligned}
$$

Making use of the Koszul's formula, we get

$$
\left\{\begin{array}{lll}
\nabla_{e_{2}} e_{3}=-\beta e_{1}, & \nabla_{e_{2}} e_{2}=0, & \nabla_{e_{2}} e_{1}=-\beta e_{3}, \\
\nabla_{e_{3}} e_{3}=0, & \nabla_{e_{3}} e_{2}=0, & \nabla_{e_{3}} e_{1}=-\beta e_{3}, \\
\nabla_{e_{1}} e_{3}=\beta e_{2}, & \nabla_{e_{1}} e_{2}=-\beta e_{3}, & \nabla_{e_{1}} e_{1}=0 .
\end{array}\right.
$$

Consequently $\left(M^{3}, \eta, \zeta, \varphi, g\right)$ is an quasi-Sasakian structure that satisfies,

$$
(\nabla \mathrm{u} \varphi) \mathrm{V}=\beta(\mathrm{g}(\mathrm{U}, \mathrm{V}) \zeta-\eta(\mathrm{V}) \mathrm{U}), \quad \nabla \mathrm{u} \zeta=-\beta \varphi \mathrm{U},
$$

where $\beta \neq 0$. Hence $\left(M^{3}, \eta, \zeta, \varphi, g\right)$ define non-cosymplectic quasi-Sasakian 3-manifold. Therefore, we find the components of curvature tensor as follows:

$$
\left\{\begin{array}{l}
\mathrm{R}\left(e_{2}, e_{3}\right) e_{3}=\beta^{2} e_{2}, \quad R\left(e_{2}, e_{3}\right) e_{1}=-\beta^{2} e_{3}, \quad R\left(e_{3}, e_{2}\right) e_{2}=\beta^{2} e_{3}, \\
R\left(e_{1}, e_{3}\right) e_{3}=\beta^{2} e_{1}, \quad R\left(e_{3}, e_{1}\right) e_{1}=\beta^{2} e_{3}, \quad R\left(e_{2}, e_{1}\right) e_{1}=\beta^{2} e_{2}-\beta^{2} e_{1}, \\
R\left(e_{1}, e_{2}\right) e_{2}=\beta^{2} e_{1}, \quad R\left(e_{1}, e_{2}\right) e_{3}=\beta^{2} e_{3}, \quad R\left(e_{3}, e_{1}\right) e_{2}=0 .
\end{array}\right.
$$


From the above we can easily evaluate the value of the Ricci tensor as follows:

$$
\left\{\begin{array}{lll}
S\left(e_{1}, e_{1}\right)=2 \beta^{2}, & S\left(e_{2}, e_{2}\right)=2 \beta^{2}, & S\left(e_{3}, e_{3}\right)=2 \beta^{2} \\
S\left(e_{1}, e_{2}\right)=0 & S\left(e_{2}, e_{3}\right)=0, & S\left(e_{2}, e_{3}\right)=0 .
\end{array}\right.
$$

Also, the scalar curvature $\mathrm{K}$ is given by:

$$
\kappa=\sum_{i=1}^{3} g\left(e_{i} e_{i}\right) S\left(e_{i}, e_{i}\right)=6 \beta^{2}
$$

Also from the Equ.(2.23), we get

$$
S\left(e_{1}, e_{1}\right)=S\left(e_{2}, e_{2}\right)=-\mu, S\left(e_{3}, e_{3}\right)=-\mu-\alpha .
$$

It is clear that $\mu=-2 \beta^{2}$ and $\alpha=0$. Thus the manifold does not admits proper $\eta$-Ricci soliton. Hence the Theorem 3.1, Theorem 4.1, Theorem 6.1 and Theorem 7.1 are verified.

Let $\left\{e_{1}, e_{2}, e_{3}\right\}$ be a basis of the tangent space at any point. For any vector $X, Y \in \chi\left(M^{2 n+1}\right)$, we have

$$
X=a_{1} e_{1}+b_{1} e_{2}+c_{1} e_{3}, Y=a_{2} e_{1}+b_{2} e_{2}+c_{2} e_{3},
$$

where $a_{i}, b_{i}, c_{i} \in \mathfrak{R} \backslash\{0\}$, for all $i=1,2,3$.

Thus $g(X, Y)=a_{1} a_{2}+b_{1} b_{2}+c_{1} c_{2}$, and $S(X, Y)=2 \beta^{2}\left\{a_{1} a_{2}+b_{1} b_{2}+c_{1} c_{2}\right\}$. Then we obtain $S(X, Y)=2 \beta^{2} g(X, Y)$, that is, the manifold $M$ is an Einstein manifold. Hence Corollary 11.3 are hold.

Remark 12.1. In this example $\beta \neq 0$ and $\mu<0$. Thus the Ricci soliton in a 3-dimensional non-cosymplectic quasi-Sasakian manifold is always shrinking. 


\section{References}

[1] Agricola, I. and Friedrich, T., Killing spinors in supergravity with 4-fluxes ,Class.Quant.Grav.20(2003),4707-4717.

[2] Agricola, I., Friedrich, T., Nagy, P.A. and Puhle, C., On the Ricci tensor in the common sector of type II,string theory, Class. Quan. Grav.,22(2005), 2569-2577.

[3] Acharya, B. S., Figueroa A-O'Farrell, Hull, M. C., and Spence, B. J., Branes at canonical singularities and holography, Adv. Theor. Math. Phys.2 (1999), 1249-1286.

[4] Blair, D. E., The theory of quasi-Sasakian structure, Journal of Differential Geom. 1(1967), 331-345.

[5] Blair, D.E.,Riemannian geometry of contact and symplectic maniifolds, Birkhauser, Boston.(2010).

[6] Barabosa, E. and Ribeiro, E. Jr., On conformal solution of the Yamabe flow, Arch., Math.,101, (2013), 73-89.

[7] Blaga, A. M., ๆ-Ricci solitons on Lorentzian para-Sasakian manifolds, Filomat. 30, 2 (2016), 489-496.

[8] Blaga, A. M., ๆ-Ricci solitons on para-Kenmotsu manifolds, Balkan J. Geom. Appl. 20 (2015), $1-13$.

[9] Calin, C. and Crasmareanu, M., From the Eisenhart problem to Ricci solitons in f-Kenmotsu manifolds, Bull. Malays. Math. Soc. 33, 3 (2010), 361-368.

[10] Cho, J. T. and Kimura, M., Ricci soliltons and real hypersurfaces in a complex space form, Tohoku Math. J.(61) (2009), no. 2, 205-212.

[11] Chaki, M. C. and Kawaguchi, T., On almost pseudo Ricci symmetric manifolds, Tensor N.S.(68)(2007), 10-14.

[12] Deshmukh, S., Alodan, H. and Al-Sodais, H., A note on Ricci soliton, Balkan J. Geom. Appl.1,16 (2011), 48-55.

[13] De, U. C. and Sarkar, A., On $\varphi$-Ricci symmetric Sasakian manifolds, Proc. Jangjeon Math. Soc.11 (2008), 47-52.

[14] Friedrich, T., and Ivanov, S.,Almost contact manifolds connection with torsion and parallel spinors, Journal Reine Angew. Math. 559, 217-236.

[15] Gray, A., Einstein-like manifolds which are not Einstein, Geom. Dedicata.7 (1978), 259-280. 
[16] Gonzalez, J. C. and Chinea, D., Quasi-Sasakian homogeneous structures on the generalized Heisenberg group $\mathrm{H}(\mathrm{p}, 1)$, Proc. Amer. Math. Soc.105 (189), 173-184.

[17] Hamilton, R. S., The Ricci flow on surfaces, Mathematics and general relativity (Santa Cruz, CA, 1986), 237-262, Contemp. Math.71, Amer. Math. Soc,Providence, RI, 1988.

[18] Hui, S. K., Yadav, S. K. and Patra, A., Almost conformal Ricci solitons on f-Kenmotsu manifolds, Khayyam J. Math. 1,5 (2019),84-104.

[19] Hui, S. K., Yadav, S. K. and Chaubey, S. K., ๆ-Ricci soliton on 3-dimensional f-Kenmotsu manifolds, Appl. Appl. Math., 13, 2 (2018), 933-951.

[20] Ivey, T., Ricci solitons on compact 3-manifolds, Diff. Geom. Appl.3 (1993), 301-307.

[21] Kanemaki, S., Quasi-Sasakian manifolds, Tohoku Math. J.29 (1977),227-233.

[22] Kim, B. H., Fibred Riemannian spaces with quasi-Sasakian structure, Hiroshima Math.J.20 (1990),477-513.

[23] Kon, M., Invariant submanifolds in Sasakian manifolds, Mathematische Annalen.219 (1976), 277-290.

[24] Mandal, A. K. and De, A., Some theorems on 3-dimensional quasi-Sasakian manifolds, Tamsui Oxford Journal of Information and Mathematical Sciences.27 (2011), 411-427.

[25] Olszak, Z., On three-dimensional conformally flat quasi-Sasakian manifolds, Period, Math. Hungar.2,33 (1996),105-113.

[26] Oubina, J. A.,New classes of almost contact metric structures, Publ. Math. Debreceen.32 (1985), 187-193.

[27] Olszak, Z., Normal almost contact metric manifolds of dimension three,Ann. Polon.Math. $47(1986), 41-50$.

[28] Pokhariyal, G. P., Yadav, S. K. and Chaubey, S. K., Ricci solitons on trans-Sasakian manifolds, Differential Geometry-Dynamical Systems.20 (2018), 138-158.

[29] Singh, H., and Khan, Q., On special weakly symmetric Riemannian manifolds, Publ. Math. Debrecen.3,58 (2001), 523-536.

[30] Tanno, S.,Quasi-Sasakian structure of rank $(2 p+1)$, Journal Differential Geom.5 (1971), 317324.

[31] Yadav, S. K., Ozturk, H., On ( $\epsilon$ )-almost paracontact metric manifolds with conformal $\eta$-Ricci solitons, Differential Geometry-Dynamical Systems. 21 (2019), 202-215. 
[32] Yadav, S. K., Chaubey, S. K. and Suthar, D. L., Some results of $\eta$-Ricci soliton on (LCS)manifolds, Surveys in Mathematics and its Applications.13 (2018), 237-250.

[33] Yadav, S. K., Chaubey, S. K. and Suthar, D. L., Some geometric properties of $\eta$-Ricci solitons and gradient Ricci solitons on $(\mathrm{LCS})_{\mathfrak{n}}$-manifolds, Cubo a Mathematical Journal, 2, 19 (2017), $33-48$.

[34] Yano, K., Integral Formulas in Riemannian geometry, Marcel Dekker, New York, 1970.

[35] Ye, R., Global existence and convergence of Yamabe flow, Journal Differ., Geom. 1, 39 (1994),35-50.

[36] Yadav, S. K., Ricci solitons on para-Kähler manifolds, Extracta Mathematicae, Available online April 29, 2, 34 (2019). 